\title{
Receding horizon control method applied to antiviral treatment of AIDS
}

\author{
A. M. Elaiw
}




\title{
RECEDING HORIZON CONTROL METHOD APPLIED TO ANTIVIRAL TREATMENT OF AIDS
}

\author{
A. M. ELAIW
}

[Received: October 23, 2003]

\begin{abstract}
This paper investigates the stabilization of a desired equilibrium point of a model describing the interaction of the immune system (CD4 cells and cytotoxic T lymphocytes CTL) with human immunodeficiecy virus HIV with sampleddata controllers. The control variable is the drug dose. Two cases of anti-HIV drugs are considered: $\mathbf{1}^{\circ}$ reverse transcriptase inhibitors (RTI), and $\mathbf{2}^{\circ}$ protease inhibitors (PI). The controller is constructed by a variant of the receding horizon control (RHC) method. We use the results obtained by [3] giving sufficient conditions which guarantee that the receding horizon controller that stabilizes the approximate discrete-time model also stabilizes the exact discrete-time model for sufficiently small discretization parameters. Results of simulations are discussed.
\end{abstract}

Mathematics Subject Classification: 93D15, 93C57, 92D30

Keywords: Receding horizon control, AIDS, feedback stabilization, sampled data systems

\section{INTRODUCTION}

Mathematical models which describe the interaction of the immune system with human immunodeficiency virus HIV have recently become the object of scientific research (see, e. g., $[\mathbf{1}, \mathbf{2}, \mathbf{4}, \mathbf{1 5}, \mathbf{1 6}])$. Anti-retroviral drug therapy has successfully been used to significantly suppress viral replication and to delay disease progression in many patients. Currently, these drugs act by two mechanisms: reverse transcriptase inhibitors (RTI) interfere with the process of reverse transcriptase and prevent the virus from infecting a cell; protease inhibitors (PI) prevent the assembly of new infectious virus by an infected cell. Optimal control theory is used to optimize the administration of drugs by considering the drug as a controller $u(t)$. One way to design an open-loop optimal controller $u^{*}(t)$ in connection with the HIV/AIDS model

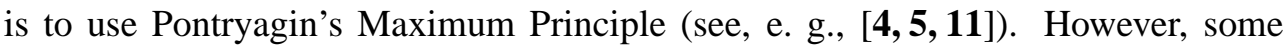
drawbacks may arise during the application of this method: $\mathbf{1}^{\circ}$ the optimization is performed over a finite time horizon, and no care is taken of the evolution of the 
process behind this time horizon; $\mathbf{2}^{\circ}$ the optimal controller is obtained as continuoustime controller in spite of the patient taking the drug doses in a discrete manner; $\mathbf{3}^{\circ}$ the optimal controller is given in open-loop form and it does not deal with the changes that may happen in the system during the treatment. Therefore, it seems to be better to design a feedback controller based on the discrete-time model.

To overcome the first mentioned problem, the application of the receding horizon method seems to be obvious. Nonlinear receding horizon control has received much interest in the academic community in the past years $[\mathbf{7 , 9 , 1 0 , 1 2 ]}$ due to the capacity of handling constraints and obtaining a stabilizing state feedback controller. Receding horizon control RHC is a form of the control in which the current control action is obtained by solving on-line, at each sampling instant, a finite horizon open-loop optimal control problem, using the current state of the plant as the initial state; the optimization yields an optimal control sequence and the first control in this sequence is applied to the plant.

As far as the second problem is concerned, one has to investigate sampled data systems. The stabilization of sampled data nonlinear systems is studied by several papers (see, e. g., $[6, \mathbf{1 3}, \mathbf{1 4}]$ ). For nonlinear systems it is unusual to assume the knowledge of the exact discrete-time model, hence we actually use the approximate discrete-time model most of the time. If we use the approximate discrete-time model, then the sampling period $T$ and/or integration parameter $h$ should be sufficiently small in order to have a small mismatch between the exact and approximate models.

Stabilization of sampled data nonlinear systems by receding horizon control via approximate discrete-time models is studied in $[\mathbf{3 , 8}]$. In these papers sufficient conditions are established which guarantee that the receding horizon controller that renders the origin to be asymptotically stable for the approximate model also stabilizes the exact discrete-time model for sufficiently small discretization parameters for the cases of fixed sampling period and varying integration parameter [3] and when these two parameters coincide [8]. The aim of this paper is to investigate the stabilization of the HIV/AIDS model by receding horizon controller in both (RTI) and (PI) cases using the results obtained in $[\mathbf{3 , 8}]$.

\section{RECEDING HORIZON CONTROL FOR SAMPLED DATA SYSTEMS}

In this section, we shall give a summary of the idea of the receding horizon control method and the main results of [3].

Consider a nonlinear control system described by

$$
\dot{x}(t)=f(x(t), u(t)),
$$

where $x(t) \in \mathbb{R}^{n}, u(t) \in U \subset \mathbb{R}^{m}, f: \mathbb{R}^{n} \times U \rightarrow \mathbb{R}^{n}$, with $f(0,0)=0, U$ is closed, and $0 \in U$. The system is to be controlled digitally using piecewise constant control functions $u(t)=u(k T)=: u_{k}$, if $t \in[k T,(k+1) T), k \in \mathbb{N}$, where $T>0$ is the sampling period. The function $f$ is assumed to have enough regularity to guarantee 
the existence and uniqueness of solutions of (1) with $u(t) \equiv \bar{u}, x(0)=x_{0}$, defined on $[0, T]$. Let $t \in[0, T] \mapsto \phi\left(t, x_{0}, \bar{u}\right)$ denote this solution. Then the exact discrete-time model of the system can be defined as

$$
x_{k+1}=F_{T}^{e}\left(x_{k}, u_{k}\right),
$$

where $F_{T}^{e}(x, u):=\phi(T, x, u)$.

We emphasize that $F_{T}^{e}$ in (2) is not known in most cases and, therefore, the controller design can be carried out by means of an approximate discrete-time model

$$
x_{k+1}=F_{T, h}^{a}\left(x_{k}, u_{k}\right) \text {. }
$$

The results of [3] and [8] cover two cases: (i) $T \neq h$, and, in this case $T$ is fixed and the family of approximate discrete-time models is generated by a numerical integration method with adjustable integration step $h$ where, $\sum_{i=0}^{m-1} h_{i}=T$ and $h_{i} \leq h$ [3]; (ii) $T=h$, and $T$ can be adjusted [8].

It is unreasonable to consider the case when $T=h$ to obtain the approximate discrete-time model for the HIV/AIDS model since, the sampling period $T$ cannot be adjusted arbitrarily. Therefore, we shall study the case when $T \neq h$ using the results obtained by [3].

We assume that both $F_{T, h}^{a}$ and $F_{T}^{e}$ are continuous in $x$ and $u$ and satisfy a local Lipschitz condition.

The problem is to define a state-feedback controller at least in a neighborhood $\overline{\mathscr{X}} \subset \mathbb{R}^{n}$ of the origin

$$
v_{T, h}^{a}: \overline{\mathscr{X}} \longrightarrow U
$$

using the approximate model (3) which practically asymptotically stabilizes the origin for the exact model (2).

Theorem $1([\mathbf{6 , 1 3}])$. Suppose that, in a given compact neighborhood of the origin, the following conditions are satisfied:

(i) the origin is an asymptotically stable equilibrium of the family of the closedloop systems $\left(F_{T, h}^{a}, v_{T, h}^{a}\right)$ with a uniformly continuous Lyapunov function $V_{T, h}^{a}(\cdot)$;

(ii) the family of controllers $v_{T, h}^{a}$ is uniformly bounded;

(iii) the family $F_{T, h}^{a}$ is consistent with $F_{T}^{e}$.

Then the family of the closed-loop systems $\left(F_{T}^{e}, v_{T, h}^{a}\right)$ is practically asymptotically stable near the origin.

Thus, a state-feedback controller $v_{T, h}^{a}$ has to be defined using the approximate model (3) for which all conditions of Theorem 1 are satisfied. In order to define such a state feedback, let equation (3) be subject to the cost function

$$
J_{T, h}^{a}(N, x, \mathbf{u})=\sum_{k=0}^{N-1} T l_{h}\left(\phi_{T, h}^{a}(k, x, \mathbf{u}), u(k)\right)+g\left(\phi_{T, h}^{a}(N, x, \mathbf{u})\right),
$$


under the constraints

$$
\begin{aligned}
u(k) & \in U, \\
\phi_{T, h}^{a}(N ; x, \mathbf{u}) & \in \mathscr{G}_{\eta}=\{x: g(x) \leq \eta\},
\end{aligned}
$$

where $l_{h}$ and $g$ are the running and terminal costs, $\mathbf{u}=\left\{u_{0}, u_{1}, \ldots, u_{N-1}\right\}$ is the control sequence and $\phi_{T, h}^{a}\left(k, x_{0}, \mathbf{u}\right)$ is the trajectory of the approximate model.

Consider the optimization problem

$$
P_{T, h}^{a}(N, x): \min \left\{J_{T, h}^{a}(N, x, \mathbf{u}), u(k) \in U, \phi_{T, h}^{a}(N ; x, \mathbf{u}) \in \mathscr{G}_{\eta}\right\} .
$$

If this optimization problem has a solution denoted by $\mathbf{u}^{*}=\left\{u_{0}^{*}, u_{1}^{*}, \ldots, u_{N-1}^{*}\right\}$, then the first element of $\mathbf{u}^{*}$, i. e., $u_{0}^{*}$ is applied at the state $x$. Since the optimal solution of $P_{T, h}^{a}(N, x)$ naturally depends on $x$, in this way a feedback has been defined on the basis of the approximate discrete-time model, i. e.,

$$
v_{T, h}^{a}(x):=u_{0}^{*} .
$$

We shall introduce the following notation

$$
V_{T, h}^{a}(x)=J_{T, h}^{a}\left(N, x, \mathbf{u}^{*}\right) .
$$

Assumption A1. The running and the terminal cost functions satisfy the following conditions:

(i) $l_{h}$ is continuous with respect to $x$ and $u$, uniformly in small $h$;

(ii) there exist $h^{*}>0$ and two class- $\mathscr{K}_{\infty}$ functions $\varphi_{1}$ and $\varphi_{2}$ such that the inequality

$$
\begin{aligned}
\varphi_{1}(\|x\|)+\varphi_{1}(\|u\|) & \leq l_{h}(x, u) \\
& \leq \varphi_{2}(\|x\|)+\varphi_{2}(\|u\|)
\end{aligned}
$$

holds for all $x, u$ and $h \in\left(0, h^{*}\right]$;

(iii) $g$ is a continuous, strictly positive definite radially unbounded function.

Assumption A2. There exist an $\eta>0$ and $h^{*}>0$ such that for all $x \in \mathscr{G}_{\eta}$ there exists a $k(x) \in U$ such that inequality

$$
\operatorname{Tl}_{h}(x, k(x))+g\left(F_{T, h}^{a}(x, k(x))\right) \leq g(x)
$$

holds true for all $h \in\left(0, h^{*}\right]$.

Assumption A3. Let $\Delta>0,0<T \leq T^{*}$ be fixed. The exact discrete-time model is practically asymptotically controllable from $\mathscr{B}_{\Delta}=\left\{x \in \mathbb{R}^{n}:\|x\| \leq \Delta\right\}$ to the origin.

Using Theorem 1, the following theorem is proven in [3].

Theorem 2 ([3]). Let us suppose that $F_{T, h}^{a}$ is consistent with $F_{T}^{e}$ and, in addition, assumptions $\mathrm{A} 1-\mathrm{A} 3$ hold. Then, in a given compact set, the family of the closed-loop systems $F_{T}^{e}$ brought about the receding horizon control (7) is practically asymptotically stable near the origin. 


\section{THE HIV/AIDS MODEL}

The mathematical model proposed by [1] and [4] is the nonlinear control system described by

$$
\begin{aligned}
& \dot{\bar{x}}(t)=\lambda-d \bar{x}(t)-\beta(t) \bar{x}(t) \bar{v}(t), \\
& \dot{\bar{y}}(t)=\beta(t) \bar{x}(t) \bar{v}(t)-a \bar{y}(t)-p \bar{y}(t) \bar{z}(t), \\
& \dot{\bar{v}}(t)=q(t) \bar{y}(t)-r \bar{v}(t), \\
& \dot{\bar{z}}(t)=c \bar{y}(t) \bar{z}(t)-b \bar{z}(t) .
\end{aligned}
$$

Here, $X(t)=(\bar{x}(t), \bar{y}(t), \bar{v}(t), \bar{z}(t))^{\prime} \in \mathbb{R}^{4}, t \in \mathbb{R}_{\geq 0}$, and the variables $\bar{x}(t), \bar{y}(t)$, $\bar{v}(t)$ and $\bar{z}(t)$ represent the uninfected CD4 cells, infected cells, free viruses, and the HIV-antigen specific cytoxic T lymphocytes (CTL). Moreover, it is assumed that the parameters $\beta$ and $q$ present an exponential characteristic with respect to controller $u$ as follows:

$\mathbf{1}^{\circ}$ The first case (RTI)

$$
\beta(t)=\beta_{0} e^{-u(t)}, \quad q=q_{0} ;
$$

$2^{\circ}$ The second case $(\mathrm{PI})$

$$
q(t)=q_{0} e^{-u(t)}, \quad \beta=\beta_{0},
$$

where $u$ is the drug dose, $u \in U \subset \mathbb{R}_{\geq 0}$ with a given closed set $U$. In the case of absence of treatments (i. e., $\beta=\beta_{0}$ and $q=q_{0}$ ), (10) leads to the model proposed by [15]. In order to avoid numerical difficulties during the integration, a normalization of the variables is introduced.

In this model the infected cells are produced by rate $\beta \bar{x} \bar{v}$ and die at rate $a \bar{y}$. Free viruses are produced from infected cells $q \bar{y}$ and decline at rate $r \bar{v}$, therefore the average life time of an infected cell is $1 / a$ and the average life time of free virus particle is $1 / r$. The total number of virus particles produced from one cell is $q / a$. Uninfected cells are produced at a constant rate $\lambda$, from a pool of precursor cells and die at rate $d x$.

The model takes into account the production of CTL by the organism, the rate of CTL proliferation in response to antigen is given by $c \bar{y} \bar{z}$ and decay at rate $b \bar{z}$, and the infected cells are killed by CTLs at rate $p \bar{y} \bar{z}$. The parameter $c$ denotes the CTL responsiveness, defined earlier as the growth rate of specific CTLs after encountering infected cells. The parameter $p$ specifies the rate at which CTLs cells kill infected cells. If $\bar{y}>b / c$, the CTL response will increase. 
The stationary points of model (10) in the absence of the drug doses, i. e., at $u=0$, are:

$$
\begin{aligned}
& X_{0}^{(1)}=\left[\frac{\lambda}{d}, 0,0,0\right] \\
& X_{0}^{(2)}=\left[\frac{a r}{q_{0} \beta_{0}}, \frac{\lambda}{a}-\frac{d r}{q_{0} \beta_{0}}, \frac{q_{0} \lambda}{a r}-\frac{d}{\beta_{0}}, 0\right] \\
& X_{0}^{(3)}=\left[\frac{c \lambda r}{b q_{0} \beta_{0}+c d r}, \frac{b}{c}, \frac{b q_{0}}{c r}, \frac{-a\left(b q_{0} \beta_{0}+c d r\right)+c q_{0} \lambda \beta_{0}}{p\left(b q_{0} \beta_{0}+c d r\right)}\right] .
\end{aligned}
$$

The matrix of the linearized system is

$$
\left(\begin{array}{cccc}
-d-\beta_{0} v_{0} & 0 & -\beta_{0} x_{0} & 0 \\
\beta_{0} v_{0} & -a-p z_{0} & \beta_{0} x_{0} & -p y_{0} \\
0 & q_{0} & -r & 0 \\
0 & c z_{0} & 0 & c y_{0}-b
\end{array}\right)
$$

where $\left(x_{0}, y_{0}, v_{0}, z_{0}\right)$ is any stationary point. Before infection the system is in the invariant hyperplane $\bar{y}=\bar{v}=\bar{z}=0$ and it has one stationary point $X_{0}^{(1)}$. If the basic reproductive ratio of model (10), $R_{0}=\frac{\lambda q_{0} \beta_{0}}{a d r}$, is smaller than 1 , then at the beginning of the infection, each virus-infected cell produces on average less than one newly infected cell. Thus, the infection cannot spread and the system returns to the uninfected state $X_{0}^{(1)}$ (stable case). If $R_{0}$ is larger than 1 , then initially each virusinfected cell produces on the average more than one newly infected cell (unstable case).

The values of parameters of the model obtained from [4] are presented in Table 1.

\begin{tabular}{|l|l|l|l|l|l|l|l|l|l|}
\hline Parameters & $d$ & $\lambda$ & $a$ & $p$ & $b$ & $c$ & $r$ & $q_{0}$ & $\beta_{0}$ \\
\hline Values & 1 & 1 & 0.8 & 0.05 & 0.01 & 0.1 & 0.01107 & 1 & 1 \\
\hline
\end{tabular}

TABLE 1

In this case, $R_{0}$ is greater than one and the stationary points become:

$$
\begin{aligned}
& X_{0}^{(1)}=[1,0,0,0] \\
& X_{0}^{(2)}=[0.008856,1.23893,111.918,0] \\
& X_{0}^{(3)}=[0.0996669,0.1,9.03342,164.067] .
\end{aligned}
$$

Since the first stationary point $X_{0}^{(1)}$ is the healthy one, we shall consider the stabilization of the system around this point. The eigenvalues of the matrix of the linearized system at $X_{0}^{(1)}$ are $\{0.669455,-1.48052,-1,-0.01\}$. 
Now we shall consider the first case (RTI) and the second case (PI) can be shown in a similar way. For convenience we transform the point $X_{0}^{(1)}$ into the origin with $x=\bar{x}-\frac{\lambda}{d}, y=\bar{y}, v=\bar{v}, z=\bar{z}$, then (10) takes the form

$$
\begin{aligned}
\dot{x}(t) & =-d x(t)-\beta_{0} e^{-u(t)}\left(x(t)+\frac{\lambda}{d}\right) v(t) \\
\dot{y}(t) & =\beta_{0} e^{-u(t)}\left(x(t)+\frac{\lambda}{d}\right) v(t)-a y(t)-p y(t) z(t) \\
\dot{v}(t) & =q_{0} y(t)-r v(t) \\
\dot{z}(t) & =c y(t) z(t)-b z(t) .
\end{aligned}
$$

Let the approximate discrete-time model be defined by the second order Taylor series method in the following way. Let $\bar{X}=\left(x^{a}(k), y^{a}(k), v^{a}(k), z^{a}(k)\right)^{\prime}, \bar{u}=u(k)$. With $\bar{X}=X_{0}$, let

$$
\begin{gathered}
x_{k+1}=x_{k}+h\left[-d x_{k}-\beta_{0} e^{-\bar{u}}\left(x_{k}+\frac{\lambda}{d}\right) v_{k}\right]+\frac{h^{2}}{2}\left[\left(-d-\beta_{0} e^{-\bar{u}} v_{k}\right)\right. \\
\left.\left(-d x_{k}-\beta_{0} e^{-\bar{u}}\left(x_{k}+\frac{\lambda}{d}\right) v_{k}\right)-\beta_{0} e^{-\bar{u}}\left(x_{k}+\frac{\lambda}{d}\right)\left(q_{0} y_{k}-r v_{k}\right)\right], \\
y_{k+1}=y_{k}+h\left[\beta_{0} e^{-\bar{u}}\left(x_{k}+\frac{\lambda}{d}\right) v_{k}-a y_{k}-p y_{k} z_{k}\right] \\
+\frac{h^{2}}{2}\left[\beta_{0} e^{-\bar{u}} v_{k}\left(-d x_{k}-\beta_{0} e^{-\bar{u}}\left(x_{k}+\frac{\lambda}{d}\right) v_{k}\right)\right. \\
-\left(a+p z_{k}\right)\left(\beta_{0} e^{-\bar{u}}\left(x_{k}+\frac{\lambda}{d}\right) v_{k}-a y_{k}-p y_{k} z_{k}\right) \\
\left.+\beta_{0} e^{-\bar{u}}\left(x_{k}+\frac{\lambda}{d}\right)\left(q_{0} y_{k}-r v_{k}\right)-p y_{k}\left(c y_{k} z_{k}-b z_{k}\right)\right] \\
v_{k+1}=v_{k}+h\left[q_{0} y_{k}-r v_{k}\right]+\frac{h^{2}}{2}\left[-r\left(q_{0} y_{k}-r v_{k}\right)\right. \\
\left.+q_{0}\left(e^{-\bar{u}}\left(x_{k}+\frac{\lambda}{d}\right) v_{k}-a y_{k}-p y_{k} z_{k}\right)\right]
\end{gathered}
$$

and

$$
\begin{aligned}
z_{k+1}=z_{k} & +h\left[c y_{k} z_{k}-b z_{k}\right]+\frac{h^{2}}{2}\left[\left(c y_{k}-b\right)\left(c y_{k} z_{k}-b z_{k}\right)\right. \\
& \left.+c z_{k}\left(\beta_{0} e^{-\bar{u}}\left(x_{k}+\frac{\lambda}{d}\right) v_{k}-a y_{k}-p y_{k} z_{k}\right)\right],
\end{aligned}
$$

$k=0,1, \ldots, m-1$, and let $X^{a}(k+1)=\left(x_{m}, y_{m}, v_{m}, z_{m}\right)^{\prime}$ where $h=T / m$. The running and the terminal costs can be chosen such that the final state constraints (5) and Assumptions A1-A2 are satisfied: 
$\mathbf{1}^{\circ}$ Case (RTI)

$\mathbf{2}^{\circ}$ Case (PI)

$$
\begin{gathered}
l_{h}(X, u)=0.01 u^{2}+x^{2}+y^{2}+(\sqrt{10} v)^{6}+z^{2} \\
g(X)=100\left(x^{2}+y^{2}+v^{4}+z^{2}\right)
\end{gathered}
$$

$$
\begin{gathered}
l_{h}(X, u)=0.001 u^{2}+x^{2}+y^{2}+1000 v^{2}+z^{2} \\
g(X)=100\left(x^{2}+y^{2}+v^{4}+z^{2}\right) .
\end{gathered}
$$

Simulations for the continuous-time system in both cases were carried out using ODE45 program in MATLAB when $h=\frac{T}{m}, T=0.5, m=50$ for $(\mathrm{RTI})$ and $T=0.5$, $m=30$ for (PI).

\section{Numerical Results}

We assume that the infection started near the equilibrium point $X_{0}^{(1)}$ with the values $\bar{x}_{0}=1, \bar{y}_{0}=0, \bar{v}_{0}=0.001$ and $\bar{z}_{0}=0.03$. For comparison purposes, we present numerical simulations of two mechanisms of treatment (RTI) and (PI) when RHC strategy is used. We vary initiation of the treatment beginning with $T_{1}$ and $T_{2}$ time units after the onset of infection (dashed and solid lines types on the Figures, respectively). Also, the non-treated case $(u=0)$ is shown (dotted lines on the Figures).

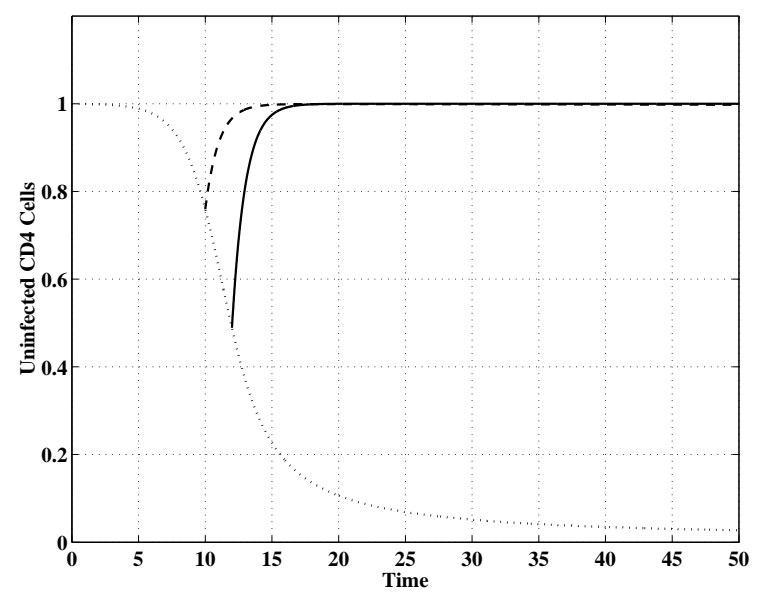

FigURe 1A

On Figures 1a and 1b, the number of uninfected CD4 cells $\bar{x}$ is seen to decay rapidly for non-treated case $(u=0)$ and tends to the value $\lambda / d$ when RHC is used. Moreover, comparing the two kinds of treatment one can observe slower convergence to the equilibrium in the (PI) case than in the (RTI) one. 


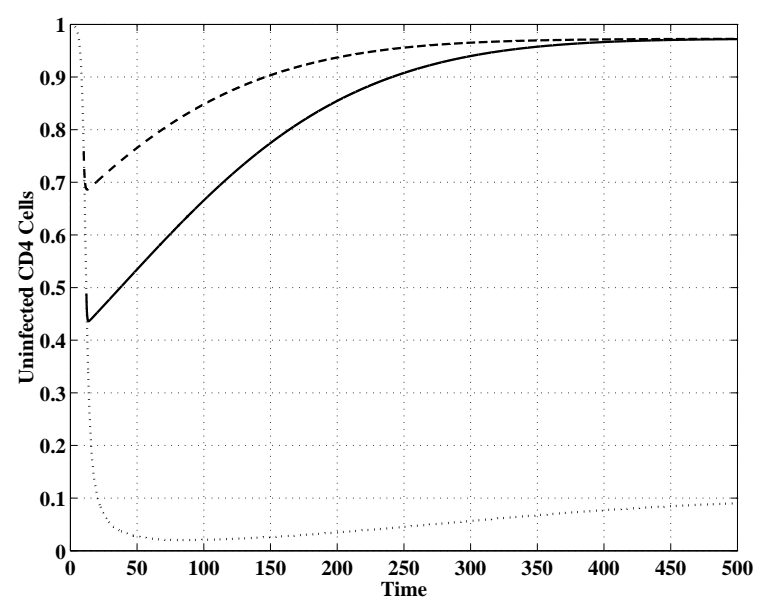

FIGURE 1B

The variation of the number of infected cells $\bar{y}$ with time is shown in Figures $2 \mathrm{a}$ and $2 \mathrm{~b}$. It is found that $\bar{y}$ increases when $u=0$, and tends to zero when RHC is applied. Further, it is seen that the convergence of $\bar{y}$ is faster in the (RTI) case than in the (PI) case.

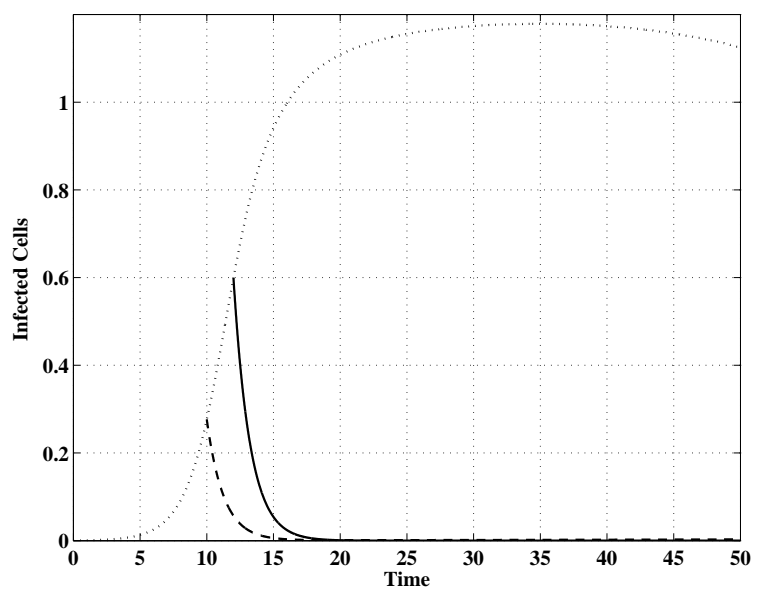

Figure 2A 


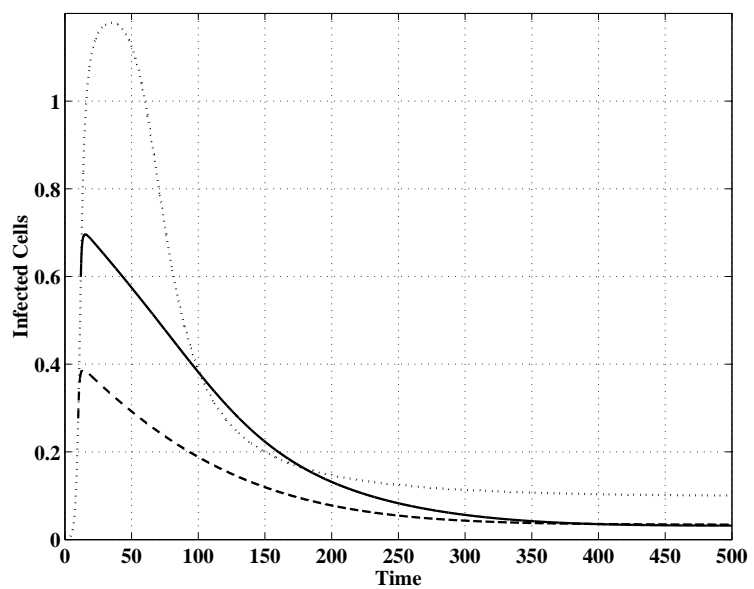

FigURE 2B

Figures $3 \mathrm{a}$ and $3 \mathrm{~b}$ show that the amount of free viruses $\bar{v}$ decreases under the application of RHC in both the (RTI) and the (PI) cases and it rapidly increases if there is no control.

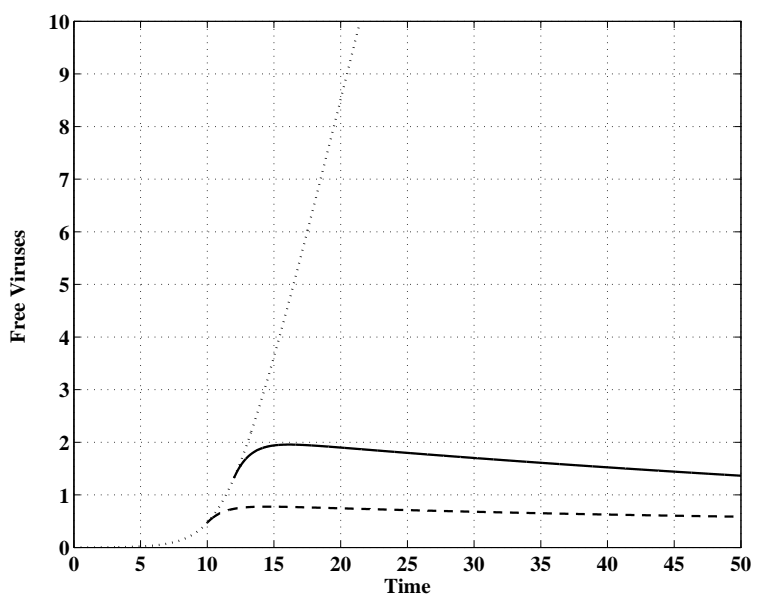

FigURe 3A

The CTL response $\bar{z}$ can be seen in Figures $4 \mathrm{a}$ and $4 \mathrm{~b}$. It is found that the amount of CTL rapidly increases for the non-treated case. Also, when the RHC is applied, 


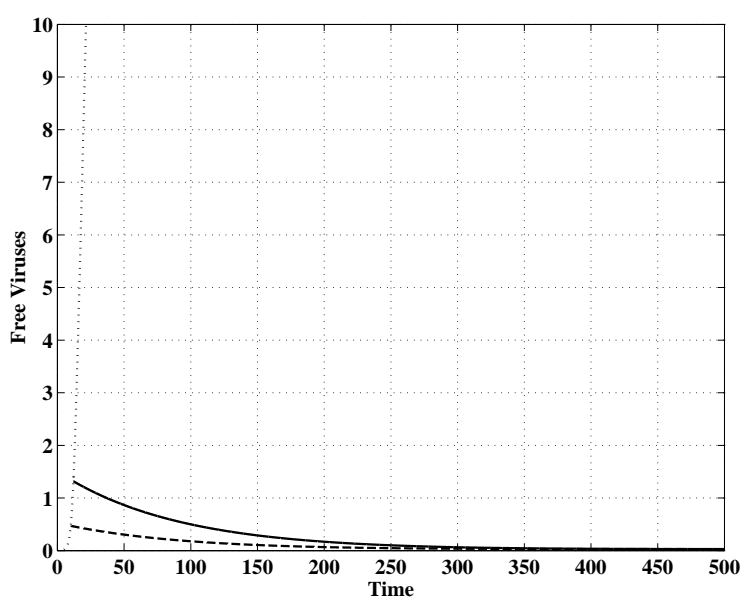

FigURE 3B

the amount of CTL increases if $\bar{y}>b / c$ and decreases if $\bar{y}<b / c$ for both the (RTI) and the $(\mathrm{PI})$ cases.

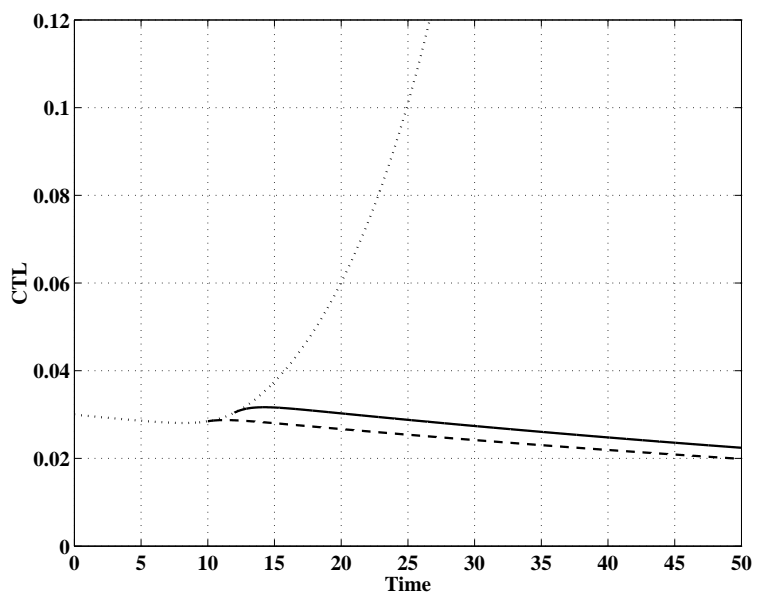

FigURe 4A

The control as a function of the time produced by RHC method for both the (RTI) and the (PI) cases is shown in Figures 5a and 5b. The total amount of drugs during finite intervals is given in Table 2 when $T_{1}=10$ and $T_{2}=12$. 


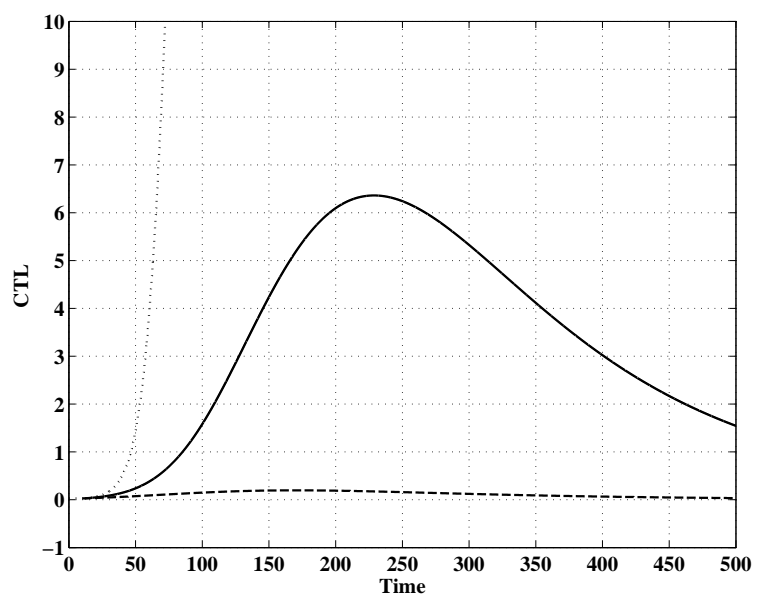

FigURe 4B

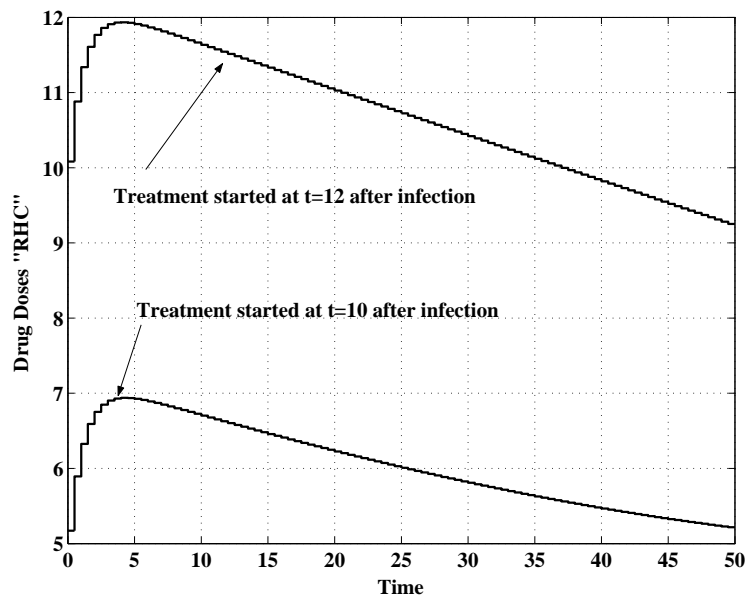

FigURE 5A

We can say that the system can be stabilized by a smaller amount of drug doses if we start the treatment earlier.

\section{Conclusions}

The stabilizing property of the RHC method for sampled data nonlinear systems via their approximate models was proven. The proposed method was applied for the 


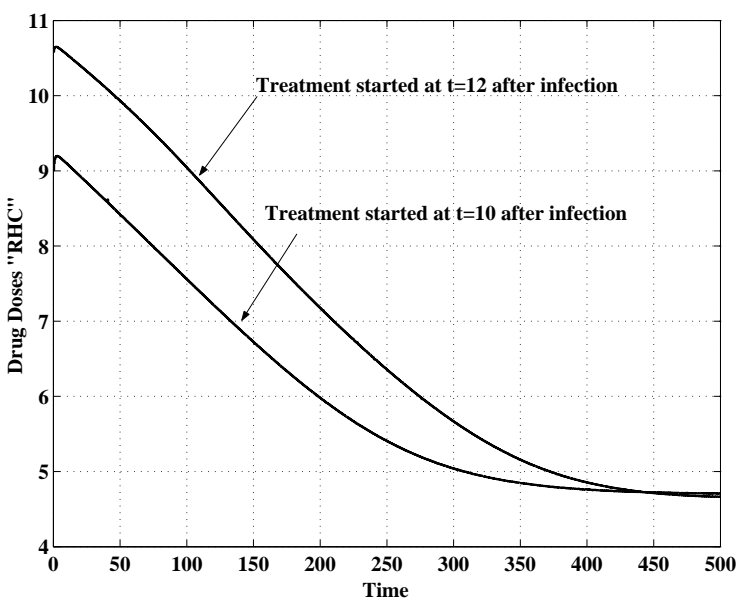

FigURE 5B

stabilization of the uninfected equilibrium point of the HIV/AIDS model. Two types of treatment were considered. The results of simulations show that the proposed method can effectively be applied to eliminate some drawbacks of the approaches previously published in the literature.

\begin{tabular}{|l|l|l|l|}
\hline Case & Interval & Total Amount of Drugs $\left(T_{1}\right)$ & Total Amount of Drugs $\left(T_{2}\right)$ \\
\hline \hline RTI & {$[0,50]$} & 603 & 1068.25 \\
\hline PI & {$[0,500]$} & 6037.5 & 6861.37 \\
\hline
\end{tabular}

TABLE 2

\section{ACKNOWLedGement}

The author is grateful to Prof. E. Gyurkovics (School of Mathematics, Budapest University of Technology and Economics) for her precious ideas and comments.

\section{REFERENCES}

[1] Caetano, M. A. L. and Yoneyama, T.: A comparative evaluation of open loop and closed loop drug administration strategies in the treatment of AIDS, Anais da Academia Brasileira de ciencias, 71 (1999), 589-597.

[2] Caetano, M. A. L., Felippe de Souza, J. A. M and Yoneyama, T.: A model based analysis of AIDS treatment, Proceedings of the Western Multiconference on Health Sciences Simulation 2003, Orlando, USA. pp. 56-70.

[3] Elaiw, A. M., and Gyurkovics, É.: Stabilizing receding horizon control of sampled-data nonlinear systems via their approximate discrete-time models, Proceedings of the $12^{\text {th }}$ IFAC Workshop on Control Applications of Optimization, Visegr'ad, Hungary (2003). 
[4] Felippe de Souza, J. A. M., Caetano, M. A. L. and Yoneyama, T.: Optimal control theory applied to the anti-viral treatment of AIDS, Proceedings of the $39^{\text {th }}$ Conference on decision and control, Sydney, Australia (2000).

[5] Fister, K. R., Lenhart, S. and McNally, J. S.: Optimizing chemotherapy in an HIV model, Electronic J. of Differential Equations, 1998 No. 32 (1998), 1-12.

[6] GRÜNE, L. and NEšić, D.: Optimization based stabilization of sampled-data nonlinear systems via their approximate discrete-time models, SIAM J. of Control Optim. 42 (2003), 98-122.

[7] Gyurkovics, É.: Receding horizon control via Bolza-type optimization, System and Control Letters, 35 (1998), 195-200.

[8] Gyurkovics, É. and Elaiw, A. M.: Stabilization of sampled data nonlinear systems by receding horizon control via discrete-time approximation, Proceedings of the $2^{\text {nd }}$ IFAC Conference on Control Systems Design, Bratislava, Slovac Republic (2003).

[9] Iто, K. and Кunisch, K.: Asymptotic properties of receding horizon optimal control problems, SIAM J. of Control Optim., 40 (2002), 1585-1610.

[10] JADABABAIE, A. and Hauser, J.: Unconstrained receding horizon control of nonlinear systems, IEEE Trans. Automatic Control, 46 (2001), 776-783.

[11] Kirschner, S., Lenhart, S. and Serbin, S.: Optimal control of the chemotherapy of HIV, J. Math. Biol. 35 (1997), 775-792.

[12] Mayne, D. Q., Rawling, J. B., Rao, C. V. and Scokaert, P. O. M.: Constrained model predictive control: Stability and optimality, Automatica, 36 (2000), 789-814.

[13] Nešić, D. and Teel, A. R.: A framework for stabilization of nonlinear sampled-data systems based on their approximate discrete-time models, IEEE Trans. Automatic Control (2003), to appear.

[14] Nešić, D., Teel, A. R. and Кокотоvić, P. V.: Sufficient conditions for stabilization of sampleddata nonlinear systems via discrete-time approximation, System and Control Letters, 38 (1999), 259-270.

[15] NowaK, M. A. and Bangham, R. M.: Population dynamics of immune responses to persistent viruses, Science, 272 (1996), 74-79.

[16] Wodarz, D. and NowaK, M. A.: Mathematical models of HIV pathogenesis and treatment, BioEssays, 24 (2002), 1178-1187.

\section{Author's Address}

\section{A. M. Elaiw:}

Budapest University of Technology and Economics, School of Mathematics, H-1521 Budapest, HuNGARY

E-mail address: elaiw@math.bme.hu 